\title{
Feed Intake, Digestibility, Growth Performance and Blood Profiles of Three Ethiopian Fat Tail Hair Sheep Fed Hay Supplemented with Two Levels of Concentrate Supplement
}

\author{
Shashie Ayele ${ }^{1,2^{*}}$, Mengistu Urge ${ }^{2}$, Getachew Animut ${ }^{3}$, Mohammed Yusuf ${ }^{2}$ \\ ${ }^{1}$ Department of Animal Sciences, College of Agriculture and Natural Resources, Debre Markos University, Debremarkos, Ethiopia \\ ${ }^{2}$ School of Animal and Range Sciences, College of Agriculture and Environmental Sciences, Haramaya University, Dire Dawa, \\ Ethiopia \\ ${ }^{3}$ Agricultural Transformation Agency, Addis Ababab, Ethiopia \\ Email: ${ }^{\star}$ aklileshashie@gmail.com
}

How to cite this paper: Ayele, S., Urge, M. Animut, G. and Yusuf, M. (2017) Feed Intake, Digestibility, Growth Performance and Blood Profiles of Three Ethiopian Fat Tail Hair Sheep Fed Hay Supplemented with Two Levels of Concentrate Supplement. Open Journal of Animal Sciences, 7, 149-167.

https://doi.org/10.4236/ojas.2017.72013

Received: February 28, 2017

Accepted: April 17, 2017

Published: April 20, 2017

Copyright $\odot 2017$ by authors and Scientific Research Publishing Inc. This work is licensed under the Creative Commons Attribution International License (CC BY 4.0).

http://creativecommons.org/licenses/by/4.0/

\begin{abstract}
An experiment arranged in $2 \times 3$ ( 2 concentrate supplement levels (CSL) and 3 breeds) factorial was carried out to evaluate the effect of CSL, breeds, and their interaction on feed intake, digestibility, feed utilization efficiency, and blood profiles of Ethiopian fat-tail hair sheep. The CSL were 1\% and 1.75\% body weight, designated as L1 and L2, respectively. The breeds used were yearling intact male Blackhead Ogaden (BHO), Horro and Washera. Sixteen sheep from each breed were divided into two based on initial body weight and randomly allocated to two dietary treatments for digestibility and growth trials of 10 and 90 days, respectively. Grass hay as a basal diet was offered ad libitum to each sheep. Feed intake every day and live body weight at interval of ten days were recorded for the duration of the experiment. Blood samples and linear body measurements were taken a week before the end and on the last day of the experiment from all sheep, respectively. Dry matter and nutrient intakes, except concentrate supplement and $\mathrm{CP}$, were influenced by the interaction. The higher $(\mathrm{p}<0.0001) \mathrm{DM}$ digestibility was recorded from sheep supplemented with L2 than L1. Consequently, sheep supplemented with L2 had significantly higher $(\mathrm{P}<0.05)$ feed conversion efficiency $(\mathrm{FCE})$, average daily gain (ADG) (57.3 vs $44.2 \mathrm{~kg}$ ), total gain (TG) (5.3 vs $3.9 \mathrm{~kg}$ ) and final body weight (FBW) than those supplemented with L1. Among breeds, Horro sheep recorded higher $(\mathrm{P}<0.05) \mathrm{DM}$ intake, digestibility, and FCE than the rest of the breeds. Consequently, this breed has significantly $(\mathrm{p}<0.05)$ higher TG, ADG, and FBW. Some of the blood hematology (PCV, MCV, neutrophils, and monocytes) and sera metabolites (total protein, urea, and cholesterol)
\end{abstract}


values were influenced by the interaction in favor of L2. Hemoglobin concentration was higher for Washera than the other breeds $(\mathrm{p}<0.05)$ but similar for the two CSL $(\mathrm{P}>0.05)$. In conclusion, the study indicate the presence of variation in nutrient intake and utilization, performance traits, and blood profiles among breeds and between CSL, most of which were in favor of Horro breed and high level of concentrate.

\section{Keywords}

Blood Hematology, Linear Body Measurement, Serum Biochemistry, Sheep

\section{Introduction}

Sheep (Ovisaries) are found across all garo-ecologies and in nearly all production systems. They are suitable for extensive to highly mechanized production systems. Globally, sheep are the species with the highest number of recorded breeds, contributing $25 \%$ to the total mammalian breeds [1], and they are used for a variety of purposes. Sheep make a substantial contribution to the wellbeing of multitudes of people around the World by providing meat, milk, fibre, and skin. Like some other countries in the world, Ethiopia possesses genetically diverse sheep [2] and it owns the second largest sheep population in Africa [3]. The sheep breeds of the country are widely adapted to different climates, ranging from sub-alpine to arid lowlands and are found in all production systems being entirely raised under traditional production system and characterized by low input-output [2] [4].

In Ethiopia, sheep plays a crucial role in the national economy, but have low growth rate with average carcass weight not more than $10 \mathrm{~kg} / \mathrm{head}$ [5] that limited the earning from the sector. The low productivity is attributed to inadequate indigenous breed utilizations for production, poor nutrition, and poor genotype [4] [6]. So far, a number of research works have been conducted to assess the effect of plane of nutrition on growth performance and carcass traits of a particular sheep breed [7] [8] [9]. However, considering the large sheep genetic resource base, comparative studies that show whether the performance of Ethiopian fat tail hair sheep is differently influenced by genotype and level of supplementation are very limited.

Various research results noted that animal growth is a function of the animal's genetic potential and the extent to which the environment, particularly nutrition, allows this potential to be expressed [10] [11]. Effect of genotype [12] [13], genotype and feeding system [14], and genotype and levels of feeding [15] on performance of sheep has been reported, although potential differences in response to different feed levels among genotypes have not been sufficiently illustrated. Knowledge of comparative growth potential among sheep genotypes and their response to different level of feeding are essential to identify the potential breed as well as to establish various management alternatives for the different 
genotypes.

In the present study, comparative performance of three sheep breeds, namely Black head Ogaden (BHO), Horro and Washera under feedlot condition was evaluated. Performance measures investigated include feed intake, digestibility, growth performance and blood profiles.

\section{Materials and Methods}

\subsection{Experimental Site}

The study was conducted during March to July 2015 at Haramaya University goat farm, located at $9^{\circ} 25^{\prime} \mathrm{N}$ latitude and $42^{\circ} 2^{\prime} \mathrm{E}$ longitude. The area lies at an altitude of 1950 meters above sea level (m.a.s.l) and receives $790 \mathrm{~mm}$ total average annual rainfall of bimodal type. It has an average temperature of $16^{\circ} \mathrm{C}$ with mean maximum and minimum annual temperatures of $24.02^{\circ} \mathrm{C}$ and $9.73^{\circ} \mathrm{C}$, respectively [16].

\subsection{Description of Sheep and Experimental Design}

Three indigenous sheep breeds, BHO, Horro and Washera were used for the comparative study. Blackhead Ogaden breed has fat rump tail [2] and inhabits the arid low land part of the country and widely distributed within $42^{\circ} \mathrm{E}-48^{\circ} \mathrm{E}$ longitudes and $3^{\circ} \mathrm{N}-9^{\circ} \mathrm{N}$ latitudes [17]. The breed is well adapted to heat, feed and water shortage and has long distance tracking ability [18] [19]. According to ESGPIP [20] BHO sheep is one of the most preferred sheep breed to Middle East export markets. Horro breed inhabits tepid to cool wet highlands in range of altitude 1400 to 2000 m.a.s.l in North West Ethiopia between $35^{\circ} \mathrm{E}$ and $38^{\circ} \mathrm{E}$ longitudes and $6^{\circ} \mathrm{N}$ and $10^{\circ} \mathrm{N}$ latitudes [17]. The breed is characterized by long fat-tail and large size and is the most prolific and best meat producing breed under good environment [18]. Washera breed is characterized by wide fat tail, large body size, long and thin legs and long neck [21]. The breed inhabits the moist highland part of the country in altitude range of 2000 to 3100 m.a.s.l in northern Ethiopia of West and East Gojam zones of the Amhara Region extending to the south of Lake Tana.

Though the university has no established system for ethical approval of animal experiments, all animal handling practices followed the international guiding principles listed by the Council for International Organizations of Medical Sciences and the International Council for Laboratory Animal Science [22].

A total of 48 (16 from each breed) yearling intact male sheep were purchased from the niche markets of the breeds and used for the experiment. Age of the animals was determined by their dentition and information obtained from the owners. The initial body weights were $19.5 \pm 0.2,22.5 \pm 0.2$ and $21.8 \pm 0.2 \mathrm{~kg}$ (mean \pm SE) for BHO, Horro and Washera breeds, respectively. Sheep were ear tagged for identification and quarantined for 21 days. During this time, they were vaccinated for ovine pasteurellosis $(2 \mathrm{ml} / \mathrm{head})$, injected with Ivermectin $(0.2 \mathrm{mg} / \mathrm{kg})$ and de-wormed with Albendazole $(15 \mathrm{mg} / \mathrm{kg})$ against endo-para- 
sites, and sprayed with Diazinine ( $1 \mathrm{ml} /$ liter of water) for ecto-parasites. At the end of the quarantine, each sheep was weighed and placed in an individual pen $(134.8 \times 78 \mathrm{~cm})$ equipped with feed trough and water bucket. The sheep were acclimated to the experimental feeds and procedures for 15 days, which was followed by 10 days of digestibility and 90 days of growth trial in that order.

The experiment was conducted in a $2 \times 3$ ( 2 CSL and 3 breeds) factorial arrangement of treatment. The 16 sheep of a breed were divided into two based on their initial body weight and randomly allocated to the two levels of concentrate supplement. The two CSL were supplementation at $1 \%$ and $1.75 \%$ body weight of each sheep, designated as L1 and L2, respectively and was provided in two equal halves at 800 and 1600 hours. The amount of supplement offer was adjusted every 10 days based on the body weight of the individual animal. The concentrate supplement comprised of wheat bran (44\%), noug cake (29\%), maize grain (26\%) and salt (1\%) on DM basis. The supplement mix is set to maximize the energy and protein content of the diet. Wheat bran is used as a cheap energy source and maize grain is included to maximize the energy content of the supplement. Dhakad et al. [23] reported lower ADG for growing lambs when wheat bran replaced cake at high level $(75 \%$ wheat bran and $22 \%$ groundnut cake) and suggested a threshold level of 50\% inclusion of wheat bran, a level that did not affect lamb growth adversely, on which the present proportion was based. Noug cake (29\%) and maize grain (26\%) is included to make metabolizable energy content of $11.94 \mathrm{MJ} / \mathrm{kg} \mathrm{DM}$ and $19.2 \% \mathrm{CP}$ to ensure maximum growth with least cost. Clean water was available to animals all the time. The hand chopped natural pasture hay basal diet was provided ad libitum at $20 \%$ refusal rate. The hay offer was adjusted every week based on their intake. The hay was dominantly composed of grass genera such as Sporobolus, Digitaria and Eragrostis and very few herbaceous legumes.

\subsection{Feed Intake and Digestibility}

At the end of adaptation period, digestibility trial was conducted using all sheep. The lambs were adapted to carry fecal collection bags for three days, which was followed by 7 consecutive days of daily total fecal output collection for each animal. About $20 \%$ of the daily total fecal output was taken to form a composite sample for each animal. The sample was stored at $-20^{\circ} \mathrm{C}$ until it was thawed, thoroughly mixed and a sub sample of about $10 \%$ was taken and kept in a deep freezer until needed for analysis.

Feed offers and refusals during the 7 days of digestibility as well as growth trial of 90 days were recorded for each animal on a daily basis and a composite feed sample per feed and refusal sample per treatment was formed for each trials. Dry matter intake was calculated as a difference between the offer and refusal. The apparent digestibility coefficient of DM and nutrients were calculated using a ratio of nutrient consumed minus nutrient in faeces over nutrient consumed multiplied by hundred. 


\subsection{Body Weight and Linear Body Measures}

The lambs were weighed at the commencement of the experiment and every 10 days thereafter. The final body weight (FBW) was taken the night before the lambs were slaughtered. All measurements were taken after overnight withdrawal of feed and water. Total gain (TG) was calculated as the difference between FBW and initial body weight (IBW). Average daily gain (ADG) was determined by dividing differences of the FBW and IBW by the number of feeding days. Feed conversion efficiency (FCE) was calculated as a proportion of ADG to daily dry matter(DM) intake.

$$
\text { Feed conversion efficiency }=\frac{\text { Average daily gain }(\mathrm{g} / \text { day })}{\text { Average daily dry matter intake }\left(\frac{\mathrm{g}}{\text { day }}\right)}
$$

The metabolize energy MJ/day intake was estimated from digestible organic matter intake (DOMI) values by using the equation of AFRC $[24]$ as $\mathrm{ME}(\mathrm{MJ} / \mathrm{d})=$ $0.0157^{\star} \mathrm{DOMI} \mathrm{g} / \mathrm{kg} \mathrm{DM}$.

At the end of the growth trial, linear body measurements such as heart girth (circumference around the chest just behind the front legs and withers), body length (the distance from base of tail to the base of the neck), height at withers (the distance from the surface of a platform on which the animal stands to the withers), pelvic width (distance between the two ends of the pelvis bone), neck circumference (circumference at base of neck), thigh circumference (circumference around the middle of the thigh) were taken from each sheep using measuring tape [25]. The measurements were taken in the morning before animals were offered feeds and water to reduce errors due to gut fill and at similar position when animals stand correctly.

\subsection{Laboratory Analysis of Feeds, Feces, and Blood Profiles}

Sub samples of feed offer, feces and refusals were dried at $60^{\circ} \mathrm{C}$ for 72 hours and ground to pass $1 \mathrm{~mm}$ sieve screen and used for chemical analysis. Dry matter content was determined by drying the samples in an oven at $105^{\circ} \mathrm{C}$ overnight while ash content was determined by burning the samples at $550^{\circ} \mathrm{C}$ for 5 hours in a muffle furnace. Nitrogen $(\mathrm{N})$ was determined by Kjeldahl method [26]. Neutral detergent fiber (NDF) and acid detergent fiber (ADF) were determined following the procedure described by Van Soest and Robertson [27].

The Packed Cell Volume (PCV), haemoglobin ( $\mathrm{Hgb}$ ), mean corpuscular volume $(\mathrm{MCV})$, mean corpuscular hemoglobin $(\mathrm{MCH})$, mean corpuscular hemoglobin concentration (MCHC), red blood cell $(\mathrm{RBC})$, total white blood cell (WBC), differential WBC (nuetrophil, lymphocyte, and monocyte), and platelet were determined from whole blood by electronic cell counter (Hematology Analyzer, 2.5 Release, Germany). Total protein was analyzed from serum by Biuret method, urea by enzymatic method (GLDH method), creatinine using photometric colorimetric test for kinetic measurements (Jaffe'-reaction), glucose using enzymatic colorimetric test (GOD-PAP method), and cholesterol level was determined using enzymatic colorimetric method with lipid clearing factor 
(CHOD-PAP method) using the reagents developed for each parameter test and the instrument Huma analyzer 3000, Germany.

\subsection{Statistical Analysis}

Data were analyzed using the general linear model (PROC GLM) procedure of SAS [28]. Adjusted Tukey test $(\mathrm{p}<0.05)$ was used to locate means that are significantly different. The statistical model used was: $Y i j k l=\mu+B i+B j+F k+(B \times$ F)jk + Eijkl. Where; Yijkl = the response variable; $\mu=$ overall mean; $B i=$ effect of block; $\mathrm{B} j=$ effect of breed; $F k=$ effect of CSL; $(B \times F) j k=$ interaction between breed and CSL, and Eijkl = random error. When the interaction effects were significant, the interaction least square means were presented and discussed. In the absence of interaction, least square means of the main effects were presented and discussed.

\section{Results}

\subsection{Chemical Composition of the Experimental Feeds}

The hay contains a CP level that can satisfy the maintenance requirement of ruminants, but high NDF and ADF content reflect late stage of maturity at harvest (Table 1). On the other hand, the concentrate mix and its ingredients had high $\mathrm{CP}$, low level of NDF and ADF values with an estimated metabolizable energy content of $11.94 \mathrm{MJ} / \mathrm{kg} \mathrm{DM}$.

\subsection{Dry Matter and Nutrient Intakes}

There was significant $(\mathrm{p}<0.001)$ breed by CSL interaction for hay, total DM, OM $(\mathrm{P}<0.0001)$, NDF $(\mathrm{P}<0.0001)$, and ME intakes $(\mathrm{P}<0.01)$ (Table 2). Generally there were differences in response to $\mathrm{DM}$ and nutrient intakes between groups consumed the two CSL, which appeared to be a possible reason for the interactions observed. Crude protein and concentrate intakes varied among genotypes and between CSL. Horro sheep consumed higher $(\mathrm{p}<0.0001)$ amount of concentrate supplement than the other breeds. Crude protein intake ranked Horro $>$ Washera $>$ BHO $(\mathrm{p}<0.0001)$. Crude protein and concentrate supplement consumption were greater at high (L2) than low level (L1) of supplement.

Table 1. Chemical composition (\% for DM and \%DM for others) of the experimental diets.

\begin{tabular}{cccccc}
\hline Diets & DM & OM & CP & NDF & ADF \\
\hline Hay & 89.7 & 92.7 & 7.9 & 79.4 & 50.2 \\
Concentrate mix & 88.8 & 94.5 & 19.2 & 39.9 & 12.8 \\
Maize grain & 86.9 & 98.6 & 8.4 & 29 & 4.2 \\
Wheat bran & 87.9 & 94.5 & 15.5 & 52.9 & 13.9 \\
Nougseed cake & 90.7 & 94.1 & 36.2 & 29.4 & 15.6
\end{tabular}

$\mathrm{DM}=$ dry matter, $\mathrm{OM}=$ organic matter, $\mathrm{CP}=$ crude protein, $\mathrm{NDF}=$ neutral detergent fiber, $\mathrm{ADF}=$ acid detergent fiber. Concentrate mix consists: maize grain (26\%), Noug cake (29\%), wheat bran (44\%) and salt $(1 \%)$. 
Table 2. Dry matter and nutrient intakes of three Ethiopian fat tail hair sheep breeds fed two concentrate supplement levels.

\begin{tabular}{|c|c|c|c|c|c|c|c|c|c|c|c|}
\hline \multirow[b]{2}{*}{ Intakes } & \multirow[b]{2}{*}{ CSL } & \multicolumn{3}{|c|}{ Breeds (B) } & \multicolumn{3}{|c|}{ CSL } & \multirow[b]{2}{*}{ SEM } & \multicolumn{3}{|c|}{$\mathrm{P}$ value } \\
\hline & & $\mathrm{BHO}$ & $\mathrm{H}$ & $\mathrm{W}$ & SEM & L1 & $\mathrm{L} 2$ & & CSL & B & $\mathrm{CSL} \times \mathrm{B}$ \\
\hline \multirow[t]{3}{*}{ Hay $(g / d)$} & & & & & & & & & 0.0113 & $<0.0001$ & 0.0002 \\
\hline & $\mathrm{L} 1$ & $440^{\mathrm{e}}$ & $548^{\mathrm{a}}$ & $480^{\mathrm{d}}$ & 9.10 & & & & & & \\
\hline & $\mathrm{L} 2$ & $509^{b c}$ & $533^{\mathrm{ab}}$ & $487^{\mathrm{cd}}$ & & & & & & & \\
\hline $\mathrm{CS}(\mathrm{g} / \mathrm{d})$ & & $205^{\mathrm{b}}$ & $244^{\mathrm{a}}$ & $237^{\mathrm{b}}$ & 3.65 & $184^{\mathrm{b}}$ & $272^{\mathrm{a}}$ & 2.98 & $<0.0001$ & $<0.0001$ & 0.0971 \\
\hline \multirow[t]{3}{*}{$\mathrm{TDM}(\mathrm{g} / \mathrm{d})$} & & & & & & & & & $<0.0001$ & $<0.0001$ & 0.0002 \\
\hline & $\mathrm{L} 1$ & $595^{\mathrm{d}}$ & $754^{\mathrm{b}}$ & $675^{\mathrm{c}}$ & 11.60 & & & & & & \\
\hline & $\mathrm{L} 2$ & $764^{\mathrm{b}}$ & $816^{\mathrm{a}}$ & $766^{\mathrm{b}}$ & & & & & & & \\
\hline \multirow[t]{3}{*}{ TDM (\%BW) } & & & & & & & & & $<0.0001$ & 0.0421 & 0.0008 \\
\hline & L1 & $2.7^{\mathrm{c}}$ & $2.9^{\mathrm{b}}$ & $2.8^{\mathrm{c}}$ & 0.03 & & & & & & \\
\hline & $\mathrm{L} 2$ & $3.0^{\mathrm{a}}$ & $3.1^{\mathrm{a}}$ & $2.9^{\mathrm{b}}$ & & & & & & & \\
\hline $\mathrm{CP}(\mathrm{g} / \mathrm{d})$ & & $83^{c}$ & $93^{\mathrm{a}}$ & $89^{\mathrm{b}}$ & 1.18 & $80.5^{\mathrm{b}}$ & $95.8^{\mathrm{a}}$ & 0.96 & $<0.0001$ & $<0.0001$ & 0.0944 \\
\hline \multirow[t]{3}{*}{$\mathrm{OM}(\mathrm{g} / \mathrm{d})$} & & & & & & & & & $<0.0001$ & $<0.0001$ & $<0.0001$ \\
\hline & $\mathrm{L} 1$ & $551^{\mathrm{d}}$ & $710^{\mathrm{b}}$ & $633^{c}$ & 11.10 & & & & & & \\
\hline & $\mathrm{L} 2$ & $723^{\mathrm{b}}$ & $769^{\mathrm{a}}$ & $723^{\mathrm{b}}$ & & & & & & & \\
\hline \multirow[t]{3}{*}{$\mathrm{NDF}(\mathrm{g} / \mathrm{d})$} & & & & & & & & & $<0.0001$ & $<0.0001$ & $<0.0001$ \\
\hline & $\mathrm{L} 1$ & $398^{\mathrm{e}}$ & $522^{\mathrm{ab}}$ & $464^{\mathrm{d}}$ & 7.50 & & & & & & \\
\hline & $\mathrm{L} 2$ & $504^{\mathrm{bc}}$ & $535^{\mathrm{a}}$ & $488^{c}$ & & & & & & & \\
\hline \multirow[t]{3}{*}{$\mathrm{ME}(\mathrm{MJ} / \mathrm{d})$} & & & & & & & & & $<0.0001$ & $<0.0001$ & 0.0057 \\
\hline & L1 & $6.1^{\mathrm{d}}$ & $8.5^{\mathrm{b}}$ & $6.9^{c}$ & 0.16 & & & & & & \\
\hline & $\mathrm{L} 2$ & $8.2^{\mathrm{b}}$ & $9.5^{\mathrm{a}}$ & $8.3^{\mathrm{b}}$ & & & & & & & \\
\hline
\end{tabular}

a,b,c,Within genotype and CSL in the same row, means with different superscript letter differ significantly $(\mathrm{p}<$ $0.05)$; $\mathrm{BHO}=$ Blackhead Ogaden, $\mathrm{H}=$ Horro, $\mathrm{W}=$ Washera, $\mathrm{L} 1=$ hay $+1 \%$ of body weight $\mathrm{CS}, \mathrm{L} 2=$ hay + $1.75 \%$ of body weight CS, CS = concentrate supplement, $\mathrm{CSL}=$ concentrate supplement level, $\mathrm{TDMI}=$ total dry matter intake, $\mathrm{ME}=$ metabolizable energy, $\mathrm{DM}=$ dry matter, $\mathrm{OM}=$ organic matter, $\mathrm{CP}=$ crude protein, $\mathrm{NDF}=$ neutral detergent fiber, $\mathrm{SEM}=$ standard error of mean.

\subsection{Apparent Dry Matter and Nutrient Digestibility}

Interaction was not significant for digestibility of DM and nutrients. But significant variation among breeds and between levels of concentrate supplement was apparent (Table 3 ). Horro sheep recorded significantly $(\mathrm{p}<0.0001)$ higher digestibility of DM and nutrients than $\mathrm{BHO}$ and Washera, while $\mathrm{BHO}$ and Washera had similar digestibility values. Digestibility values were significantly higher at L2 than L1 CSL.

\subsection{Body Weight and Feed Conversion Efficiency}

Breeds and concentrate levels differ significantly for all body weight parameters with the exception of initial BW for the two level of supplementation (Table 4). Horro sheep has higher ( $\mathrm{p}<0.0001)$ FBW, TG, and ADG than BHO and Washera breeds. Blackhead Ogaden sheep has higher total gain (TG) and ADG than Washera, which also has lower $(\mathrm{p}<0.0001)$ FCE than BHO and Horro breeds. Growth 
Table 3. Dry matter and nutrient apparent digestibility of three Ethiopian fat tail hair sheep breeds fed two levels of concentrate supplement.

\begin{tabular}{ccccccccccc}
\hline \multirow{2}{*}{ Digestibility \% } & \multicolumn{4}{c}{ Breeds (B) } & \multicolumn{4}{c}{ CSL } & \multicolumn{3}{c}{ P value } \\
\cline { 2 - 10 } & BHO & H & W & SEM & L1 & L2 & SEM & CSL & B & CSL $\times$ B \\
\hline DM & $69.1^{\mathrm{b}}$ & $74.8^{\mathrm{a}}$ & $67.9^{\mathrm{b}}$ & 0.46 & $68.8^{\mathrm{b}}$ & $72.4^{\mathrm{a}}$ & 0.37 & $<0.0001$ & $<0.0001$ & 0.883 \\
OM & $71.9^{\mathrm{b}}$ & $77.3^{\mathrm{a}}$ & $71.0^{\mathrm{b}}$ & 0.49 & $71.6^{\mathrm{b}}$ & $75.1^{\mathrm{a}}$ & 0.40 & $<0.0001$ & $<0.0001$ & 0.779 \\
CP & $79.9^{\mathrm{b}}$ & $83.3^{\mathrm{a}}$ & $78.8^{\mathrm{b}}$ & 0.48 & $79.6^{\mathrm{b}}$ & $81.7^{\mathrm{a}}$ & 0.39 & 0.0009 & $<0.0001$ & 0.084 \\
NDF & $67.6^{\mathrm{b}}$ & $73.8^{\mathrm{a}}$ & $65.6^{\mathrm{b}}$ & 0.76 & $67.6^{\mathrm{b}}$ & $70.4^{\mathrm{a}}$ & 0.62 & 0.0028 & $<0.0001$ & 0.209 \\
ADF & $58.4^{\mathrm{b}}$ & $65.9^{\mathrm{a}}$ & $56.8^{\mathrm{b}}$ & 0.59 & $63.2^{\mathrm{b}}$ & $65.5^{\mathrm{a}}$ & 0.48 & 0.0022 & $<0.0001$ & 0.292 \\
\hline
\end{tabular}

${ }^{a, b}$ Within genotype and CSL in the same row, means with different superscript letter differ significantly $(\mathrm{p}<$ $0.05) ; \mathrm{BHO}=$ Blackhead Ogaden, $\mathrm{H}=$ Horro, $\mathrm{W}=$ Washera, $\mathrm{L} 1=$ hay $+1 \%$ of body weight CS, L2 = hay + $1.75 \%$ of body weight CS, CS = concentrate supplement, $\mathrm{CSL}=$ concentrate supplement level, $\mathrm{DM}=$ dry matter, $\mathrm{OM}=$ organic matter, $\mathrm{CP}=$ crude protein, $\mathrm{NDF}=$ neutral detergent fiber, $\mathrm{ADF}=$ acid detergent fiber, $\mathrm{SEM}=$ standard error of mean

Table 4. Body weight and feed conversion efficiency of three Ethiopian fat tail hair sheep breeds fed two levels of concentrate supplement.

\begin{tabular}{ccccccccccc}
\hline & \multicolumn{3}{c}{ Breeds (B) } & \multicolumn{4}{c}{ CSL } & \multicolumn{3}{c}{ P value } \\
\hline Parameters & BHO & H & W & SEM & L1 & L2 & SEM & CSL & B & CSL $\times$ B \\
\hline IBW (kg) & $19.5^{\mathrm{b}}$ & $22.5^{\mathrm{a}}$ & $21.8^{\mathrm{a}}$ & 0.21 & 21.1 & 21.3 & 0.17 & 0.2036 & $<0.0001$ & 0.0556 \\
FBW (kg) & $23.9^{\mathrm{c}}$ & $27.9^{\mathrm{a}}$ & $25.7^{\mathrm{b}}$ & 0.26 & $25.1^{\mathrm{b}}$ & $26.5^{\mathrm{a}}$ & 0.21 & $<0.0001$ & $<0.0001$ & 0.3405 \\
TG (kg) & $4.4^{\mathrm{b}}$ & $5.4^{\mathrm{a}}$ & $4.0^{\mathrm{c}}$ & 0.12 & $4.0^{\mathrm{b}}$ & $5.3^{\mathrm{a}}$ & 0.09 & $<0.0001$ & $<0.0001$ & 0.665 \\
ADG (g/d) & $49.2^{\mathrm{b}}$ & $59.8^{\mathrm{a}}$ & $43.3^{\mathrm{c}}$ & 1.19 & $44.2^{\mathrm{b}}$ & $57.3^{\mathrm{a}}$ & 0.98 & $<0.0001$ & $<0.0001$ & 0.3903 \\
FCE & $0.073^{\mathrm{a}}$ & $0.076^{\mathrm{a}}$ & $0.059^{\mathrm{b}}$ & 0.001 & $0.065^{\mathrm{b}}$ & $0.074^{\mathrm{a}}$ & 0.001 & 0.0003 & $<0.0001$ & 0.0786
\end{tabular}

${ }^{\mathrm{a}, \mathrm{b}, \mathrm{c}} \mathrm{W}$ ithin breed and CSL in the same row, means with different superscript letter differ significantly $(\mathrm{p}<$ 0.05). $\mathrm{BHO}=$ Blackhead Ogaden, $\mathrm{H}=$ Horro, $\mathrm{W}=$ Washera, $\mathrm{L} 1=$ hay $+1 \%$ of body weight CS, $\mathrm{L} 2=$ hay + $1.75 \%$ of body weight CS, CS = concentrate supplement, $\mathrm{CSL}=$ concentrate supplement level, IBW = initial body weight, FBW = final body weight, $\mathrm{ADG}=$ average daily gain, $\mathrm{TG}=$ total gain, $\mathrm{FCE}=$ feed conversion efficiency (g ADG/g DMI/d), SEM = standard error of mean.

performance and feed efficiency of all the breeds were better at high than low level of concentrate supplement.

\subsection{Linear Body Measures}

With the exception of heart girth (HG) for which there was interaction ( $\mathrm{p}<$ 0.001) between breeds and levels of concentrate supplement (Table 5), only main effects were significantly variable for the other linear body measures. The HG of BHO sheep was lower ( $<0.0001)$ than Horro, while the value for Washera was similar $(p>0.05)$ with the two genotypes at the high CSL, but differ with BHO sheep at low CSL. Wither height (WH) was similar between Horro and Washera, and was higher than BHO sheep $(\mathrm{p}<0.0001)$. Pelvic width $(\mathrm{PW})$ ranked Horro $>\mathrm{BHO}>$ Washera $(\mathrm{p}<0.001)$. Neck and thigh circumference were similar between $\mathrm{BHO}$ and Washera and higher for Horro than the two breeds $(\mathrm{p}<$ 0.001). Body length (BL), PW, and thigh circumference (TC) of the sheep were higher at higher level of concentrate supplement, but WH and neck circumference (NC) were not influenced by the level of concentrate supplementation. 
Table 5. Linear body measurements of three Ethiopian fat tail hair sheep breeds fed two levels of concentrate supplement.

\begin{tabular}{|c|c|c|c|c|c|c|c|c|c|c|c|}
\hline \multirow{2}{*}{$\begin{array}{c}\text { Measurements } \\
(\mathrm{cm})\end{array}$} & \multicolumn{5}{|c|}{ Breeds (B) } & \multicolumn{3}{|l|}{ CSL } & \multicolumn{3}{|c|}{$P$ value } \\
\hline & CSL & $\mathrm{BHO}$ & $\mathrm{H}$ & $\mathrm{W}$ & SEM & L1 & $\mathrm{L} 2$ & SEM & CSL & B & $\mathrm{CSL} \times \mathrm{B}$ \\
\hline \multirow[t]{3}{*}{ Heart girth } & & & & & & & & & $<0.0001$ & $<0.0001$ & $<0.0001$ \\
\hline & L1 & $65.6^{c}$ & $69.9^{\mathrm{b}}$ & $69.5^{\mathrm{b}}$ & 0.37 & & & & & & \\
\hline & $\mathrm{L} 2$ & $69.9^{\mathrm{b}}$ & $71^{\mathrm{a}}$ & $70.3^{\mathrm{ab}}$ & & & & & & & \\
\hline Body length & & $51.4^{\mathrm{c}}$ & $57.0^{\mathrm{a}}$ & $53.9^{\mathrm{b}}$ & 0.40 & $53.4^{\mathrm{b}}$ & $54.8^{\mathrm{a}}$ & 0.30 & 0.006 & $<0.0001$ & 0.7554 \\
\hline Wither height & & $56.8^{\mathrm{b}}$ & $64.6^{\mathrm{a}}$ & $65.6^{\mathrm{a}}$ & 0.37 & $62^{\mathrm{a}}$ & $62.8^{\mathrm{a}}$ & 0.30 & 0.071 & $<0.0001$ & 0.1302 \\
\hline Pelvic width & & $17.5^{\mathrm{b}}$ & $18.3^{\mathrm{a}}$ & $16.9^{c}$ & 0.21 & $16.9^{\mathrm{b}}$ & $18.3^{\mathrm{a}}$ & 0.17 & $<0.0001$ & 0.0001 & 0.1179 \\
\hline Neck circumference & & $35.3^{\mathrm{b}}$ & $37.5^{\mathrm{a}}$ & $35.6^{\mathrm{b}}$ & 0.34 & $35.8^{\mathrm{a}}$ & $36.6^{\mathrm{a}}$ & 0.28 & 0.0683 & 0.0001 & 0.0637 \\
\hline Thigh circumference & & $15.6^{\mathrm{b}}$ & $16.6^{\mathrm{a}}$ & $15.8^{\mathrm{b}}$ & 0.16 & $15.6^{\mathrm{b}}$ & $16.4^{\mathrm{a}}$ & 0.10 & 0.0004 & 0.0006 & 0.1778 \\
\hline
\end{tabular}

\subsection{Blood Hematology}

There was an interaction effect between breed and CSL for PCV, MCV, neutrophils, and monocytes (Table 6). The interaction for PCV may be due to the low value recorded for $\mathrm{BHO}$ at the low level of concentrate supplement as compared to the other values. The MCV (PCV/RBC) increased for Washera, but decreased for Horro and $\mathrm{BHO}$ at high level of supplementation $(\mathrm{P}<0.001)$ which is an attribute of higher PCV for Washera and RBC for BHO. Neutrophils for BHO and Washera increased significantly with level of supplement, but was similar for Horro sheep. Monocyte was significantly higher at higher than low level of supplement for only BHO. Washera breed has showed higher hemoglobin concentration than $\mathrm{BHO}$ sheep. $\mathrm{BHO}$ has lower, while Horro and Washera recorded higher $\mathrm{MCH}(\mathrm{P}<0.05)$. The $\mathrm{BHO}$ sheep recorded the highest $\mathrm{RBC}$, Washera intermediate, and Horro the smallest $(\mathrm{P}<0.01)$. Horro sheep has more lymphocytes than $\mathrm{BHO}$ and Washera sheep. Mean corpuscular hemoglobin concentration, WBC, and platelet were not influenced $(\mathrm{P}>0.05)$ by both factors. Sheep consumed L1 concentrate supplement had significantly $(\mathrm{p}<0.0001)$ higher RBC than $\mathrm{L} 2$ group. Significantly $(\mathrm{P}<0.01)$ higher $\mathrm{MCH}$ and lymphocyte values were observed at L2 than L1 CSL.

\subsection{Serum Biochemistry}

There were significant CSL by breed interactions for total protein $(\mathrm{P}<0.0001)$, urea $(\mathrm{P}<0.001)$, and cholesterol $(\mathrm{P}<0.01)$ values, which were higher at higher than lower level of concentrate supplement for Horro and Washera sheep (Table 7). Nevertheless, protein and cholesterol for BHO did not defer between the two levels of concentrate supplement. Creatinine level did not differ among breeds and between CSL, but glucose was higher for BHO followed by Washera and Horro in that order $(\mathrm{P}<0.0001)$, as well as in L2 than in L1 supplemented lambs $(\mathrm{p}<0.01)$. 
Table 6. Blood hematology of three Ethiopian fat tail hair sheep breeds fed two levels of concentrate supplement.

\begin{tabular}{|c|c|c|c|c|c|c|c|c|c|c|c|c|}
\hline \multirow[b]{2}{*}{ Parameters } & \multirow[b]{2}{*}{ CSL } & \multicolumn{3}{|c|}{ Breeds (B) } & \multicolumn{3}{|c|}{ CSL } & \multicolumn{2}{|r|}{${ }^{\star}$ Reference } & \multicolumn{3}{|c|}{$\mathrm{p}$ value } \\
\hline & & $\mathrm{BHO}$ & $\mathrm{H}$ & $\mathrm{W}$ & SEM & $\mathrm{L} 1$ & $\mathrm{~L} 2$ & SEM & values & CSL & B & $\mathrm{CSL} \times \mathrm{B}$ \\
\hline \multirow[t]{3}{*}{ PCV (\%) } & & & & & & & & & & 0.1082 & 0.0001 & 0.0001 \\
\hline & L1 & $35.0^{c}$ & $36.6^{\mathrm{b}}$ & $37.4^{\mathrm{ab}}$ & 0.35 & & & & $27 \%-45 \%$ & & & \\
\hline & $\mathrm{L} 2$ & $36.6^{\mathrm{b}}$ & $36.9^{\mathrm{ab}}$ & $37.9^{\mathrm{a}}$ & & & & & & & & \\
\hline $\mathrm{Hb}(\mathrm{g} / \mathrm{dl})$ & & $10.8^{\mathrm{b}}$ & $10.9^{\mathrm{ab}}$ & $11.1^{\mathrm{a}}$ & 0.09 & 11.0 & 11.0 & 0.08 & $9-15 \mathrm{~g} / \mathrm{dl}$ & 0.2237 & 0.033 & 0.736 \\
\hline \multirow[t]{3}{*}{$\operatorname{MCV}(\mathrm{fl})$} & & & & & & & & & & 0.0003 & $<0.0001$ & $<0.0001$ \\
\hline & L1 & $27.0^{c}$ & $29.7^{\mathrm{a}}$ & $28.2^{\mathrm{b}}$ & 0.26 & & & & $20-40 \mathrm{fl}$ & & & \\
\hline & L2 & $25.7^{\mathrm{d}}$ & $27.4^{\mathrm{c}}$ & $29.0^{\mathrm{a}}$ & & & & & & & & \\
\hline $\mathrm{MCH}(\mathrm{pq})$ & & $7.9^{\mathrm{b}}$ & $8.49^{\mathrm{a}}$ & $8.5^{\mathrm{a}}$ & 0.16 & $8.0^{\mathrm{b}}$ & $8.5^{\mathrm{a}}$ & 0.13 & $8-12 p q$ & 0.0075 & 0.027 & 0.353 \\
\hline $\mathrm{MCHC}(\mathrm{g} / \mathrm{dl})$ & & 30.0 & 29.8 & 29.7 & 0.26 & 30.0 & 30.0 & 0.21 & $31-34 \mathrm{~g} / \mathrm{dl}$ & 0.965 & 0.581 & 0.156 \\
\hline $\mathrm{RBC}(\times 10)^{6} / \mu \mathrm{l}$ & & $13.7^{\mathrm{a}}$ & $12.9^{c}$ & $13.3^{\mathrm{b}}$ & 0.14 & $13.8^{\mathrm{a}}$ & $12.8^{\mathrm{b}}$ & 0.11 & $9-15\left(10^{6}\right) / \mu \mathrm{l}$ & $<0.0001$ & 0.001 & 0.713 \\
\hline $\operatorname{WBC}(10)^{3} / \mu \mathrm{l}$ & & 11.1 & 11.5 & 10.6 & 0.23 & 11.0 & 11.0 & 0.19 & $4-12\left(10^{3}\right) / \mu \mathrm{l}$ & 0.7324 & 0.064 & 0.237 \\
\hline \multirow[t]{3}{*}{ Neut. $(10)^{3} / \mu \mathrm{l}$} & & & & & & & & & & 0.0002 & 0.008 & 0.0309 \\
\hline & L1 & $5.5^{\mathrm{b}}$ & $5.4^{\mathrm{b}}$ & $4.7^{\mathrm{c}}$ & 0.13 & & & & $700-6000 / \mu \mathrm{l}$ & & & \\
\hline & L2 & $5.8^{\mathrm{a}}$ & $5.5^{\mathrm{ab}}$ & $5.6^{\mathrm{ab}}$ & & & & & & & & \\
\hline Lym. $(10)^{3} / \mu \mathrm{l}$ & & $4.9^{\mathrm{b}}$ & $5.4^{\mathrm{a}}$ & $4.7^{\mathrm{b}}$ & 0.09 & $4.8^{\mathrm{b}}$ & $5.2^{\mathrm{a}}$ & 0.08 & $(2-9) \times 10^{3} / \mu \mathrm{l}$ & 0.0087 & 0.0003 & 0.519 \\
\hline \multirow[t]{3}{*}{ Mon. $(10)^{3} / \mu \mathrm{l}$} & & & & & & & & & & 0.0001 & 0.003 & 0.043 \\
\hline & L1 & $0.32^{\mathrm{c}}$ & $0.52^{\mathrm{b}}$ & $0.68^{\mathrm{ab}}$ & 0.06 & & & & $0-750 / \mu \mathrm{l}$ & & & \\
\hline & $\mathrm{L} 2$ & $0.72^{\mathrm{a}}$ & $0.66^{\mathrm{ab}}$ & $0.8^{\mathrm{a}}$ & & & & & & & & \\
\hline Plt. $(10)^{3} / \mu \mathrm{l}$ & & 330 & 338.5 & 307.4 & 12.7 & 314 & 337 & 10.4 & & 0.1363 & 0.229 & 0.225 \\
\hline
\end{tabular}

${ }_{\mathrm{a}, \mathrm{b}, \mathrm{c}}$ Within genotype and CSL in the same row, means with different superscript letter differ significantly $(\mathrm{p}<0.05), \mathrm{BHO}=\mathrm{Blackhead} \mathrm{Ogaden}, \mathrm{H}=\mathrm{Horro}$, W $=$ Washera, $\mathrm{L} 1=$ hay $+1 \%$ of body weight CS, L2 = hay $+1.75 \%$ of body weight CS, CS = concentrate supplement, CSL $=$ concentrate supplement level, SEM $=$ standard error of mean, $\mathrm{fL}$, femtoliter, $\mathrm{mg} / \mathrm{dL}=$ mile gram per deciliter, $\mu \mathrm{l}=$ micro liter, $\mathrm{Hgb}=$ hemoglobin, $\mathrm{MCV}=\mathrm{mean}$ corpuscular volume, $\mathrm{MCH}=$ mean corpuscular hemoglobin, $\mathrm{MCHC}=$ mean corpuscular hemoglobin concentration, $\mathrm{PCV}=$ packed cell volume, $\mathrm{RBC}=$ red blood cells, $\mathrm{WBC}=$ white blood cells, Neutr $=$ Neutrophils, Lym $=$ lymphocytes, Mon $=$ Monocytes, Plt. = platelets. ${ }^{\star}$ Reference value [29].

\section{Discussion}

\subsection{Feed Intake and Digestibility}

The lack of substitution effect of concentrate supplement for hay at higher level of supplement feeding indicates that the two CSL have complementary effect on the basal diet hay [29]. Balanced concentrate supplement promotes greater roughage intake, total feed intake, and nutrient digestion and utilization [31] [32]. The difference in total DM intake (\%BW) of the three breeds may reflect variation in volume of the reticulo-rumen among the breeds. Difference in the ability to digest feed, the variation in the ADG, and other characteristics associated with animal's ability to utilize energy from the diets [33]. As noted by various researchers, the ruminants' reticulo-rumen volume determines the potential physical intake of forages [33] [34] [35]. According to Getahun [36] the level of intake and the weight of the empty reticulo-rumen in goats have positive correlation. Similar to the present study, Dereje et al. [37] observed higher intake 
Table 7. Some serum biochemistry of three Ethiopian fat tail hair sheep breeds fed two levels of concentrate supplement.

\begin{tabular}{|c|c|c|c|c|c|c|c|c|c|c|c|c|}
\hline \multirow[b]{3}{*}{ Parameters } & \multirow[b]{3}{*}{ CSL } & & \multicolumn{10}{|c|}{ Reference } \\
\hline & & & \multicolumn{2}{|c|}{ Breeds } & \multicolumn{4}{|c|}{ CSL } & \multirow{2}{*}{$\begin{array}{l}\text { values } \\
\text { SEM }\end{array}$} & \multicolumn{3}{|c|}{$P$ value } \\
\hline & & $\mathrm{BHO}$ & $\mathrm{H}$ & $\mathrm{W}$ & SEM & L1 & $\mathrm{L} 2$ & & & CSL & B & $\mathrm{CSL} \times \mathrm{B}$ \\
\hline \multirow[t]{3}{*}{ Total protein $(\mathrm{g} / \mathrm{L})$} & & & & & & & & & & $<0.0001$ & $<0.0001$ & $<0.0001$ \\
\hline & L1 & $63.6^{\mathrm{a}}$ & $58.1^{\mathrm{b}}$ & $59.4^{\mathrm{b}}$ & 0.88 & & & & $60-79 \mathrm{~g} / \mathrm{L}$ & & & \\
\hline & L2 & $74.4^{\mathrm{a}}$ & $72.4^{\mathrm{a}}$ & $73.2^{\mathrm{a}}$ & & & & & & & & \\
\hline \multirow[t]{3}{*}{ Urea $(\mathrm{mmol} / \mathrm{L})$} & & & & & & & & & & $<0.0001$ & 0.0161 & 0.0008 \\
\hline & L1 & $5.8^{\mathrm{c}}$ & $5.5^{\mathrm{d}}$ & $5.4^{\mathrm{d}}$ & 0.54 & & & & ${ }^{+} 2.86-7.14 \mathrm{mmol} / \mathrm{L}$ & & & \\
\hline & L2 & $6.1^{\mathrm{b}}$ & $6.6^{\mathrm{a}}$ & $6.2^{\mathrm{b}}$ & & & & & & & & \\
\hline Creatinine (mg/dl) & & 1.0 & 1.1 & 1.1 & 0.03 & 1.0 & 1.0 & 0.02 & $1.2-1.9 \mathrm{~g} / \mathrm{dl}$ & 0.8474 & 0.2772 & 0.3636 \\
\hline \multirow[t]{3}{*}{ Cholostrol (mmol/L) } & & & & & & & & & & $<0.0001$ & $<0.0001$ & 0.0046 \\
\hline & L1 & $1.5^{\mathrm{a}}$ & $1.2^{\mathrm{c}}$ & $1.1^{\mathrm{d}}$ & 0.62 & & & & $1.05-1.5 \mathrm{mmol} / \mathrm{L}$ & & & \\
\hline & $\mathrm{L} 2$ & $1.6^{\mathrm{a}}$ & $1.4^{\mathrm{b}}$ & $1.2^{\mathrm{c}}$ & 0.62 & & & & & & & \\
\hline Glucose $\mathrm{mmol} / \mathrm{L}$ & & $3.4^{\mathrm{a}}$ & $2.9^{c}$ & $3.0^{\mathrm{b}}$ & 0.03 & $3.0^{\mathrm{b}}$ & $3.2^{\mathrm{a}}$ & 0.50 & $2.8-4.4 \mathrm{mmol} / \mathrm{L}$ & 0.0019 & $<0.0001$ & 0.6492 \\
\hline
\end{tabular}

a,b,c, Within genotype and CSL in the same row, means with different superscript letter differ significantly $(\mathrm{p}<0.05)$; BHO = Blackhead Ogaden, $\mathrm{H}=\mathrm{Horro}, \mathrm{W}=$ Washera, $\mathrm{L} 1=$ hay $+1 \%$ of body weight CS, $\mathrm{L} 2=$ hay $+1.75 \%$ of body weight CS, CS = concentrate supplement, CSL $=$ concentrate supplement level; SEM $=$ standard error of mean, $\mathrm{mg} / \mathrm{dL}=$ mile gram per deciliter, $\mathrm{mmol} / \mathrm{L}=$ mile mole per liter, Reference values [29] [30].

at $1.5 \%$ than $1 \%$ of body weight of concentrate supplement in a trial comparing three goat breeds. Significant breed effect on total DM intake is also reported by Tsegay et al. [38]. The review work of Pulina et al. [39] showed that voluntary feed intake in sheep is transmitted from parent to offspring with $h^{2}$ value of 0.11 to 0.66 .

Higher nutrient digestion and better FCE by Horro breed may be due to higher $\mathrm{CP}$ and ME intake than BHO and Washera breeds. Likewise, L2 concentrate supplement provides higher $\mathrm{CP}$ and ME thus, higher digestibility and FCE was recorded in lambs supplemented by the higher level of concentrate supplement. As stated by McDonald et al. [40] supplementation of poor quality forage with good protein feed has increased the availability of nitrogen in the rumen, thereby improving the rate of degradation and utilization of the feed.

\subsection{Growth Performance}

In previous studies, ADG of 25 - $34 \mathrm{~g} / \mathrm{d}$ [41], 73.7 - $91.3 \mathrm{~g} / \mathrm{d}$ [8] for Washera; $47.3 \mathrm{~g} / \mathrm{d}$ [34], 40 - $93.75 \mathrm{~g} / \mathrm{d}$ [42] for Horro and $60.4-95.6 \mathrm{~g} / \mathrm{d}$ [43] for BHO that consumed basal diets and supplement with different types were reported. The variation in ADG recorded for the three breeds under different experiment is an attribute of the feeding level and diets used and the age of the animal during the experiment. In the current study, Horro breed gained more than the other two breeds which may be an attribute of better efficiency in nutrient digestion and utilization. Various researchers noted positive and strong correlation between digestibility to weight gain [7] [8] [41]. The differences in ADG between supplement levels is an attribute of higher CP and ME intake that allows more mi- 
crobial population growth and therefore promote digestion making nutrient available to increase weight gain in sheep supplemented with high level of concentrate. These findings are in agreement with that reported by Tsegay et al. [38] who observed higher ADG for the sheep supplemented with $350 \mathrm{~g} / \mathrm{d}$ concentrate than those supplemented with $150 \mathrm{~g} / \mathrm{d}$. Dereje et al. [37] also obtained higher ADG in goats supplemented with $1.5 \%$ body weight concentrate than those consumed at $1 \%$ body weight.

The difference in linear body measurements of sheep in the present study reflects morphological variation among breeds. The fact that Horro breed has higher value of hearth girth (HG), body length (BL), pelvic width (PW), thigh circumference (TC) and neck circumference (NC) indicate the breed is of large frame with a potential of gaining more body weight. As reported by Oke and Ogbonnaya [44] BL, wither height (WH), HG and NC have strong correlation with body weight in three age ( $0-12,13-24$, and $>25$ months) categories studied in Wad sheep. Vargas et al. [45] also indicated that HG, WH and BL are closely related to weight of an animal. On the other hand, Washera sheep has the highest WH since this breed has long and thin legs, and long neck as compared the other sheep breeds of Ethiopia [2] [21]. The difference in linear body measurements between supplement groups may be largely attributed to the higher $\mathrm{CP}$ and $\mathrm{ME}$ intake that enhanced skeletal growth.

\subsection{Blood Hematology}

All blood hematology and serum biochemistry values obtained for all breeds in the present study were within the normal reference range reported by Radostits et al. [29] and Kaneko et al. [30]. The marked increase in PCV in BHO at high level of concentrate supplement is attributable to the variation in the number of RBC or circulating plasma volume [46]. Variability in PCV among breeds was reported by various researchers in sheep [47] [48] and in goat [49]. The lack of effect between CSL on PCV in the present study is in line with that reported by Okah et al. [50] for west African Dwarf sheep supplemented with different levels of poultry droppings (10\%, and $20 \%$ ). Lack of difference for Hgb concentration between supplement levels is in agreement with other study conducted using Santa Inês lambs fed various mixture of physic nut meal with soybean meal and ground corn [51]. Increased level of Hgb concentration is attributed to ability to tolerate infection and good nutritional status as well as breed of animals [47] [48] [49].

The higher RBC and lower Hgb concentrations in BHO may indicate the need for higher oxygen supply for rapid cellular metabolism with increased availability of nutrients. This breed is adapted to arid environment where feed supply is scarce and may adjust their metabolic activity in accordance with feed availability, one of which could be increasing rate of metabolism which in turn requires higher oxygen supply through increased red blood cell concentration. In the present study, higher RBC was obtained at L1 than L2 CSL. Since more red blood cell count is expected at high level of supplement because of more iron 
supply, the higher RBC obtained at L1 concentrate supplement is unexplained.

The MCV, MCH, and MCHC values coupled with PCV and Hgb are very important in the diagnosis of anemia and also serve as a useful index of the capacity of the bone marrow to produce red blood cells in animals [46]. Previous findings noted that these parameters are dependent on genotype, sex, age, season [47] [49] and feed [52]. The lower MCV for BHO at both level of supplementation compared to Horro and Washera was due to higher RBC while higher $\mathrm{MCH}$ in the Horro and Washera breeds compared to $\mathrm{BHO}$ was attributed to the higher $\mathrm{Hgb}$ and lower RBC record.

The numerical difference in WBC count among sheep breeds may reflect the existence of variations in degree of disease resistance and variation in adaptability to local environmental and disease prevalence conditions [53]. Among the sheep breeds compared in the present study, Horro breed had numerically higher WBC count than Washera sheep. Tibbo [4] noted Horro sheep express their growth potential regardless of exposure to the climatic stressors and health challenges such as cold and pneumonia than the Menz sheep breed in the environment native to Menz sheep. This may indicate the superiority of the breed to tolerate diseases and to adapt to the environment to which they are exposed. In the present study, WBC differentials were significantly affected by breed, CSL, and some interaction. The effects of genotype on these parameters are in agreement with that noted by Tibbo et al. [51] and Njidda et al. [47]. The finding that diet has no substantial effects on blood platelets concord with Oliveira et al. [51] who observed similar result for Santa Inês lambs fed various mixture of physic nut meal with soya bean meal and ground corn. Lack of significant difference among genotypes on platelets contradicts with those of Jawasreh et al. [54] who obtained significant breed effect for platelets on different genetic lines of Awassi ewes. It is known that blood platelets are implicated in blood clotting. Low platelet concentration suggests that the process of clot-formation (blood clotting) will be prolonged resulting in excessive loss of blood in case of injury.

\subsection{Serum Biochemistry}

The observed total protein values in the present study are slightly lower than the values ( 64 to $83 \mathrm{~g} / \mathrm{L}$ ) reported by Njidda et al. [47] for the three breeds of Nigerian sheep. The observation that higher level of concentrate supplemented group has higher total protein than the low level group could be attributed to higher protein intake from the higher level concentrate supplement. In agreement to this study, Hoffman et al. [55] found more serum total protein and albumin but similar albumin to globulin ratio for Holstein heifers fed a diet having $15 \% \mathrm{CP}$ than $8 \% \mathrm{CP}$. Contrary to this finding, Okah et al. [50] obtained similar serum total protein concentration irrespective of dietary levels.

In the present study, BHO breed has significantly $(\mathrm{p}<0.001)$ higher value of urea than Horro and Washera at L1 concentrate supplement, which could be the attribute of higher total serum protein. Similarly, at L2 concentrate supplement, Horro breed has higher serum urea concentration than $\mathrm{BHO}$ and Washera 
breeds, which is in accordance with the crude protein intake. Lack of significant dietary effect on creatinine concentration is contrary to those of Shaker et al. [56] and Tope et al. [52]. On the other hand, lack of breed effect on this parameter disagrees with the report of Njidda et al. [47] but concurs with result of Jawasreh et al. [54]. The observed Cholesterol values in the present study are similar to 1.05 to $1.5 \mathrm{mmol} / \mathrm{L}$ documented as normal value for sheep [29] but lower than 1.8 to $2.5 \mathrm{mmol} / \mathrm{L}$ reported for yearling Northern Nigeria sheep breeds [47]. In addition, significant breed effect on cholesterol level was also observed in the work of Jawasreh et al. [54]. On the other hand, similar to these findings, significant dietary effects on cholesterol levels have been reported by Tope et al. [52] and Shaker et al. [56].

The reason for variation in glucose concentration among genotypes in the present study is the greater difference in dietary energy intake and due to variation in growing ability. Likewise, the higher glucose recorded from L2 sheep than from L1 sheep could be due to the higher dietary energy intake. Similar to the present study, Catunda et al. [57] found higher glucose concentration in supplemented hair sheep than un supplemented ones.

\section{Conclusion}

The study show that genotypes and levels of concentrate supplement influenced feed intake, growth rate, and blood profiles of the selected fat tail hair sheep breeds of Ethiopia. Concentrate supplement feeding at the rate of $1.75 \%$ than $1 \%$ body weight improved rate of digestion, dry matter intake, efficient utilization of the feed, linear body measurements and daily gain. Among breeds evaluated, Horro sheep has better dry matter intake, nutrient digestion, feed efficiency, and growth ability. This indicates the potential of the breed for meat production. Blood profile results showed variation among breeds and were highly sensitive to genotype by CSL interaction implicating that such factors need to be critically considered in the establishment of reference ranges of blood profile for Ethiopia sheep breeds. In general, the study indicated presence of variation among Ethiopian sheep breeds in growth ability and high concentrate supplement is needed to express their genetic potential.

\section{Acknowledgements}

The first author would like to thank Haramaya University for providing the financial and research facilities and Debremarkos University for providing study leave and paying salary.

\section{References}

[1] FAO (2000) Food and Agriculture Organization. World Watch List for Domestic Animal Diversity. 3rd Edition, Rome.

[2] Solomon, G., Van Arendonk Johan, A.M., Komen, H., Windig, J.J. and Hanotte, O. (2007) Population Structure, Genetic Variation and Morphological Diversity in Indigenous Sheep of Ethiopia. Animal Genetics, 38, 621-628. 
https://doi.org/10.1111/j.1365-2052.2007.01659.x

[3] FAO (2013)Food and Agriculture Organization. World Statistical Compendium for Raw Hides And Skins, Leather and Leather Footwear 1993-2012. Trade and Markets Division Food and Agriculture Organization of the United Nations.

[4] Tibbo, M. (2006) Productivity and Health of Indigenous Sheep Breeds and Crossbreds in the Central Ethiopian Highlands. PhD Dissertation, Swedish University of Agricultural Sciences, Uppsala, Sweden.

[5] FAO (1996) Food and Agriculture Organization. Improving Food Security, the Ignored Contribution of Livestock. FAO Yearbook, Production 1995, Vol. 49.

[6] Aschalew, T. (2006) Assessment of Feeding Systems and Evaluation of Feed Supplementation on Body Weight and Fleece Production of Sheep in Ethiopia. PhD Dissertation, Kasetsart University, Thailand.

[7] Fentie, B. and Solomon, M. (2008) Effects of Supplementation of Farta Sheep Fed Hay with Sole or Mixtures of Noug Seed Meal and Wheat Bran on Feed Intake, Digestibility and Body Weight Change. Tropical Animal Health and Production, 40, 597-606. https://doi.org/10.1007/s11250-008-9138-1

[8] Likawent, Y., Claudia, K., Firew, T. and Kurt, J.P. (2012) Sweet Blue Lupin (Lupinus angustifolius L.) Seed as a Substitute for Concentrate Mix Supplement in the Diets of Yearling Washera Rams Fed on Natural Pasture Hay as Basal Diet in Ethiopia. Tropical Animal Health and Production, 44, 1255-1261.

https://doi.org/10.1007/s11250-011-0066-0

[9] Birhanu, T., Getachew, A. and Mengistu, U. (2013) Effect of Green Prosopis juliflora Abissynica Cake Supplementation on Digestibility and Performance of Blackhead Ogaden Sheep Fed Hay as a Basal Diet. Science, Technology and Arts Research Journal, 2, 38-47.

[10] Oddy, V.H. and Sainz, R.D. (2002) Nutrition for Sheep-Meat Production. In: Freer, M. and Dove, H., CSIRO Plant Industry Canberra Australia, Eds., Sheep Nutrition, CABI Publishing, CAB international, Oxon, UK, 237-263. https://doi.org/10.1079/9780851995953.0237

[11] Solaiman, S.G. (2010) Goat Science and Production. John Wiley and Sons, Inc., Publication, Blackwell Publishing, Hoboken.

[12] Abdullah, Y.A., Rami, T.K., Momani, M.S. and Mohammad, D.O. (2010) Investigation of Growth and Carcass Characteristics of Pure and Crossbred Awassi Lambs. Small Ruminant Research, 94,167-175.

[13] Boujenane, I. (2015) Growth at Fattening and Carcass Characteristics of D'man, Sardi and Meat-Sire Crossbred Lambs Slaughtered at Two Stages of Maturity. Tropical Animal Health and Production, 47, 1363-1371. https://doi.org/10.1007/s11250-015-0872-x

[14] Santos-Silva, J., Mendes, I.A. and Bessa, R.J.B. (2002) The Effect of Genotype, Feeding System and Slaughter Weight on the Quality of Light Lambs. 1. Growth, Carcass Composition and Meat Quality. Livestock Production Science, 76, 17-25.

[15] Fozooni, R. and Zamiri, M.J. (2007) Relationships between Chemical Composition of Meat from Carcass Cuts and the Whole Carcass in Iranian Fat Tailed Sheep as Affected by Breed and Feeding Level. Iranian Journal of Veterinary Research, 8.

[16] Mishra, B.B., Gebrekidan, H., Kibret, K., Assen, M. and Eshetu, B. (2004) Soil and Land Resource Inventory at Alemaya University Research Farm with Reference to Land Evaluation for Sustainable Agricultural Management and Production. Synthesis of Working Papers, Soil Science Bulletin No. 1. Alemaya University, Ethiopia.

[17] Kasahun, A. and Solomon, A. (2008) Breeds of Sheep and Goats. In: Yami, A. and 
Merkel, R.C., Eds., Sheep and Goat Production Handbook of Ethiopia. ESGPIP (Ethiopian Sheep and Goat Productivity Improvement Program), Branna Printing Enterprise, Addis Ababa, Ethiopia, 5-26.

[18] Gizaw, S., Komen, H., Windig, J.J., Hanotte, O. and van Arendonk, J.A.M. (2008) Conservation Priorities for Ethiopian Sheep Breeds Combining Threat Status, Breed Merits and Contributions to Genetic Diversity. Genetics Selection Evolution, 40, 433-447. https://doi.org/10.1051/gse:2008012

[19] ESGPIP (2009) Ethiopia Sheep and Goat Productivity Improvement Program. Estimation of Weight and Age of Sheep and Goats. Technical Bulletin No. 23.

[20] ESGPIP (2011) Ethiopia Sheep and Goat Productivity Improvement Program. Export Requirements for Meat and Live Small Ruminants: How Can Development Agents Assist Producers to Improve Small Ruminant Export? Technical Bulletin No. 47.

[21] Sisay, L. (2009) Phenotypic Characterization of Indigenous Sheep Breeds in the Amhara National Regional State of Ethiopia. MSc Thesis, Haramaya University, Ethiopia.

[22] Council for International Organizations of Medical Sciences and the International Council for Laboratory Animal Science (2012) International Guiding Principles for Biomedical Research Involving Animals. http://grants.nih.gov/grants/olaw/Guiding_Principles_2012.pdf

[23] Dhakad, A., Garg, A.K., Singh, P. and Agrawal, D.K. (2002) Effect of Replacement of Maize Grain with Wheat Bran on the Performance of Growing Lambs. Small Ruminant Research, 43, 227-234. https://doi.org/10.1016/S0921-4488(02)00025-1

[24] AFRC (1993) Agricultural Food and Research Council. Energy and Protein Requirements of Ruminants. An Advisory Manual Prepared by the Agricultural Food and Research Council Technical Committee on Responses to Nutrients. CAB International, Wallingford, UK.

[25] De Boer, H., Dumont, B.L., Fomeroy, R.W. and Weniger, J.H. (1974) Manual on E.A.A.P. Reference Methods for the Assessment of Carcass Characteristics in Cattle. Livestock Production Science, 1, 151-164. https://doi.org/10.1016/0301-6226(74)90055-4

[26] AOAC (1990) Association of Official Analytical Chemists. Official Methods of Analysis. 15th Edition, AOAC, Virginia, USA.

[27] Van Soest, P.J. and Robertson, J.B. (1985) Analysis of Forages and Fibrous Foods a Laboratory Manual for Animal Science. Cornell University, Ithaca, NY.

[28] SAS (2008) Statistical Analysis Systems Institute. Version 9.1, SAS Institute Inc., Cary, North Carolina, USA.

[29] Radostits, O.M., Clive, G., Kenneth, W.H. and Peter, D.C. (2006) Veterinary Medicine: A Textbook of the Disease of Cattle, Sheep, Goats, Pigs and Horses. Saunders, Edinburgh.

[30] Kaneko, J.J., John, W.H. and Michael, L.B. (2008) Clinical Biochemistry of Domestic Animals. 6th Edition, Elsevier Inc., Amsterdam.

[31] Dove, H. (2002) Principles of Supplementary Feeding in Sheep-Grazing Systems. In: Freer, M. and Dove, H., CSIRO Plant Industry Canberra Australia, Eds., Sheep Nutrition, CABI Publishing, CAB International, Oxon, UK, 119-142.

https://doi.org/10.1079/9780851995953.0119

[32] ARC (1980) Agricultural Research Council. The Nutrient Requirements of Ruminant Livestock. Technical Review by an Agricultural Research Council Working Party, Common wealth Agricultural Bureau, Farnham Royal, UK. 
[33] Forbes, J.M. (1995) Voluntary Food Intake and Diet Selection in Farm Animals. CAB International, Oxon, UK.

[34] Kasahun, A. (2000) Comparative Performance Evaluation of Horro and Menz Sheep of Ethiopia under Grazing and Intensive Feeding Conditions. PhD Dissertation, Humboldt University, Berlin.

[35] Fisher, D.S. (2002) A Review of a Few Key Factors Regulating Voluntary Feed Intake in Ruminants. Crop Science, 42, 1651-1655. https://doi.org/10.2135/cropsci2002.1651

[36] Getahun, L. (2001) Growth Pattern and Carcass Characteristics of Somali and Mid Rift Valley Goats. MSc Thesis, Alemaya University of Agriculture, Ethiopia.

[37] Dereje, T., Mengistu, U., Getachew, A. and Yoseph, M. (2016) Growth and Carcass Characteristics of Three Ethiopian Indigenous Goats Fed Concentrate at Different Supplementation Levels. SpringerPlus, 5, 414. https://doi.org/10.1186/s40064-016-2055-2

[38] Tsegay, T., Yoseph, M. and Mengistu, U. (2013) Comparative Evaluation of Growth and Carcass Traits of Indigenous and Crossbred (Dorper $\times$ Indigenous) Ethiopian Sheep. Small Ruminant Research, 114, 247-252. https://doi.org/10.1016/j.smallrumres.2013.07.003

[39] Pulina, G., Avondo, M., Molle, G., Francesconi, A.H.D., Atzori, A.S. and Cannas, A. (2013) Models for Estimating Feed Intake in Small Ruminants. Revista Brasileira de Zootecnia, 42, 675-690. https://doi.org/10.1590/s1516-35982013000900010

[40] McDonald, P., Edwards, R.A., Greenhalgh, J.F.D., Morgan, C.A., Sinclair, L.A. and Wilkinson, R.G. (2010) Animal Nutrition. 7th Edition

[41] Hailu, A., Melaku, S., Tamir, B. and Tassew, A. (2011) Body Weight and Carcass Characteristics of Washera Sheep Fed Urea Treated Rice Straw Supplemented with Graded Levels of Concentrate Mix. Livestock Research for Rural Development, 23, Article No. 164. http://www.lrrd.org/lrrd23/8/hail23164.htm

[42] Firisa, W., Adugna, T. and Diriba, D. (2013) Feed Intake, Digestibility and Growth of Horrolambs Fed Natural Pasture Hay Supplemented with Graded Level of Vernonia amygdalina Leaves and Sorghum Grain Mixture. Science, Technology and Arts Research Journal, 2, 30-37.

[43] Wogenie, B. (2007) Effects of Increasing Levels of Energy and Protein Supplementation on Feed Intake, Body Weight Change and Carcass Composition of Blackhead Somali Sheep Fed on Grass Hay. MSc Thesis, Haramaya University, Ethiopia.

[44] Oke, U.K. and Ogbonnaya, E.O. (2011) Application of Physical Body Traits in the Assessment of Breed and Performance of WAD Sheep in a Humid Tropical Environment. Livestock Research for Rural Development, 23, Article No. 24. http://www.lrrd.org/lrrd23/2/oke23024.htm

[45] Vargas, S., Labri, A. and Sanchez, M. (2007) Analysis of Size and Conformation of Native Creole Goat Breeds and Crossbreds Used in Smallholder Agrosilvopastoral Systems in Puebla, Mexico. Tropical Animal Health and Production, 39, 276-286. https://doi.org/10.1007/s11250-007-9012-6

[46] Chineke, C.A., Ologun, A.G. and Ikeobi, C.O.N. (2006) Haematological Parameters in Rabbit Breeds and Crosses in Humid Tropics. Pakistan Journal of Biological Sciences, 9, 2102-2106. https://doi.org/10.3923/pjbs.2006.2102.2106

[47] Njidda, A.A., Shuaibu, A.A. and Isidahomen, C.E. (2014) Haematological and Serum Biochemical Indices of Sheep in Semi-Arid Environment of Northern Nigeria. Global Journal of Science Frontier Research: Division Agriculture and Veterinary, 14, 48-56. 
[48] Anwar, M.M., Nour El-Din, A.N.M. and Taha, T.A. (2012) Changes in Some Hematological and Serum Biochemical Parameters during the First Week after Lambing in Six Consecutive Parities in Some Egyptian Sheep Breeds. Egyptian Journal of Animal Production, 49, 293-302.

[49] Tibbo, M., Jibril, Y., Woldemeskel, M., Dawo, F., Aragaw, K. and Rege, J.E.O. (2004) Factors Affecting Hematological Profiles in three Ethiopian Indigenous Goat Breeds. International Journal of Applied Research Veterinary Medicine, 2, 297-309.

[50] Okah, U., Chibueze, C.O. and Anya, M.I. (2013) Haematological and Biochemical Characteristics of West African Dwarf Rams Fed Different Levels of Poultry Droppings/Maggots Combination. Journal of Agricultural Technology, 9, 1151-1156.

[51] Oliveira, P.B., Lima, P.M.T., Campeche, A., Mendonc, S., McManus, C., Louvandini, H. and Laviola, B.G. (2013) Growth and Carcass Characteristics of Santa Inês Lambs Fed Diet Supplemented with Physic Nut Meal Free of Phorbol Ester. Small Ruminant Research, 114, 20-25. https://doi.org/10.1016/j.smallrumres.2013.05.007

[52] Tope, A.F., Funmi, O., Adewumi, A. and Sunday, D.E. (2012) Performance, Hematology and Serum Biochemistry of West African Dwarf Goats Fed Ensiled Mixtures of Elephant Grass (Pennisetum purpureum) with Lima Bean, African Yam Bean and Pigeon Pea. Kasetsart Journal-Natural Science, 46, 694-702.

[53] Isaac, L.J., Abah, G., Akpan, B. and Ekaette, I.U. (2013) Haematological Properties of Different Breeds and Sexes of Rabbits. Proceeding of the 18 th Annual Conference of Nigerian Society of Animal Science, 24-27.

[54] Jawasreh, K., Awawdeh, F., Bani Ismail, Z., Al-Rawashdeh, O. and Al-Majali, A. (2013) Normal Hematology and Selected Serum Biochemical Values in Different Genetic Lines of Awassi Ewes in Jordan. The Internet Journal of Veterinary Medicine, 7.

[55] Hoffman, P.C., Esser, N.M., Bauman, L.M., Denzine, S.L., Engstrom, M. and Chester-Jones, H. (2001) Short Communication: Effect of Dietary Protein on Growth and Nitrogen Balance of Holstein Heifers. Journal of Dairy Science, 84, 843-847. https://doi.org/10.3168/jds.S0022-0302(01)74542-0

[56] Shaker, Y.M., Ibrahim, N.H., Younis, F.E. and El-Shaer, H.M. (2014) Effect of Feeding Some Salt Tolerant Fodder Shrubs Mixture on Physiological Performance of Shami Goats in Southern Sinai, Egypt. Journal of American Science, 10.

[57] Catunda, A.G.V., Lima, I.C.S., Bandeira, G.C., Gadelha, C.R.F., Salmito-Vanderley, C.S.B., Araújo, A.A., Martins, G.A., Pereira, E.S. and Campos, A.C.N. (2013) Blood Leptin, Insulin and Glucose Concentrations in Hair Sheep Raised in a Tropical Climate. Small Ruminant Research, 114, 272-279.

https://doi.org/10.1016/j.smallrumres.2013.07.008 


\section{Abbreviations}

$\mathrm{ADG}=$ average daily gain, $\mathrm{BHO}=$ Blackhead Ogaden, $\mathrm{CP}=$ crude protein, $\mathrm{CS}=$ concentrate supplement, $\mathrm{CSL}=$ concentrate supplement levels, $\mathrm{FCE}=$ feed conversion efficiency, FBW = final body weight, IBW= initial body weight, $\mathrm{DM}=$ dry matter, $\mathrm{OM}=$ organic matter, $\mathrm{NDF}=$ neutral detergent fiber, $\mathrm{ADF}=$ acid detergent fiber, $\mathrm{MCV}=$ mean corpuscular volume, $\mathrm{TG}=$ total gain, $\mathrm{PCV}=$ packed cell volume, $\mathrm{Hgb}=$ haemoglobin, $\mathrm{MCH}=$ mean corpuscular hemoglobin, $\mathrm{MCHC}=$ mean corpuscular hemoglobin concentration, $\mathrm{RBC}=$ red blood cell, $\mathrm{WBC}=$ white blood cell.

Submit or recommend next manuscript to SCIRP and we will provide best service for you:

Accepting pre-submission inquiries through Email, Facebook, LinkedIn, Twitter, etc. A wide selection of journals (inclusive of 9 subjects, more than 200 journals)

Providing 24-hour high-quality service

User-friendly online submission system

Fair and swift peer-review system

Efficient typesetting and proofreading procedure

Display of the result of downloads and visits, as well as the number of cited articles Maximum dissemination of your research work

Submit your manuscript at: http://papersubmission.scirp.org/

Or contact ojas@scirp.org 